\title{
The Relationship Between Friction and Film Thickness in EHD Point Contacts in the Presence of Longitudinal Roughness
}

\author{
Johan Guegan $^{1}$ (D) Amir Kadiric ${ }^{1} \cdot$ Antonio Gabelli $^{2} \cdot$ Hugh Spikes $^{1}$
}

Received: 18 July 2016/ Accepted: 5 October 2016

(c) The Author(s) 2016. This article is published with open access at Springerlink.com

\begin{abstract}
This study investigates friction and film thickness in elastohydrodynamic contacts of machined, rough surfaces, where roughness is dominated by longitudinal ridges parallel to the rolling/sliding direction. A ball-ondisc tribometer was used to simultaneously measure friction and film thickness in rough contacts as well as with nominally smooth specimens for comparison. The studied rough surfaces were selected so that the influence of the root-mean-square roughness and roughness wavelength can be assessed. Friction and film measurements were taken over a range of slide-roll ratios and speeds and with two lubricating oils with different viscosities, hence covering a wide range of specific film thicknesses. The measurements with the nominally smooth specimens show that friction is strongly influenced by thermal effects at high SRRs and that the transition from mixed/boundary to full EHD lubrication occurs at lambda ratios greater than three. At low speeds, the rough specimens are found to generate higher friction than the smooth ones for all the roughness structures considered, and this is shown to be related to the thinner minimum film thickness. Comparison of friction in rough and smooth contacts shows that the total friction in rough contacts can be divided into two components: one that is equivalent to friction in smooth contacts under the same conditions and is dependent on the slide-roll ratio, and the other that is due to the presence of roughness and is independent of the slide-roll ratio under the conditions
\end{abstract}

Johan Guegan

j.guegan11@imperial.ac.uk

1 Tribology Group, Department of Mechanical Engineering, Imperial College London, London SW7 2AZ, UK

2 SKF Engineering \& Research Center B.V., Nieuwegein, The Netherlands tested. Further analysis of the minimum film thickness on tops of roughness ridges indicates that even after the full lift-off, an effect of the roughness on friction persists and is most likely related to the local shear stress in the microEHD contacts on the top of roughness ridges. At even higher speeds, the difference in friction between the rough and smooth specimens vanishes.

Keywords Elastohydrodynamic lubrication · Mixed lubrication · Micro-EHL surface roughness $\cdot$ Friction

\section{Introduction}

The current trend towards the use of less viscous lubricants with the aim of reducing hydrodynamic friction is resulting in machine elements operating under ever decreasing lubricant film thicknesses. Consequently, such elements are operating for long periods in the mixed rather than the fullfilm hydrodynamic or elastohydrodynamic (EHD) lubrication regimes. In mixed lubrication, the load is shared between the lubricant film and the asperity contacts. While the effect of roughness on lubricant film thickness in nonconforming, EHD contacts such as those in gears and rolling bearings has been the subject of a number of experimental and theoretical studies (e.g. [1-3]), its influence on EHD friction has been less widely studied.

In full-film EHD contacts, where the film thickness is much greater than the surface roughness, the EHD friction originates solely from the bulk rheological properties of the lubricant at the very high pressures and strain rates present. At relatively low strain rates, the imposition of a high pressure results in a very large increase in lubricant viscosity, but at higher strain rates such viscosities cannot be maintained and all organic-based liquid lubricants exhibit 
considerable shear thinning and, thus, a greatly reduced effective viscosity. Various equations have been proposed to describe the relationship between shear stress $\tau$ and strain rate $\dot{\gamma}$ under EHD conditions one of which is the Eyring model:

$\tau=\tau_{\mathrm{E}} \sinh ^{-1}\left(\frac{\eta(P) \dot{\gamma}}{\tau_{\mathrm{E}}}\right)$

where $\eta(P)$ is the Newtonian or low shear rate dynamic viscosity (as a function of the pressure $P$ ) and $\tau_{\mathrm{E}}$ is the Eyring stress above which the lubricant starts to shear thin. The total friction force in the EHD contact is then simply the integral of the shear stress over the area of the contact.

At high shear stresses and strain rates present in EHD, considerable lubricant shear heating may also occur which will reduce $\eta(P)$ and hence the shear stress and friction force [4].

Some authors $[5,6]$ have considered the influence of random roughness on friction. In these studies, the roughness was characterized solely by the composite root-meansquare roughness of the surfaces, RMS, defined as:

$\mathrm{RMS}=\sqrt{R_{q 1}{ }^{2}+R_{q 2}{ }^{2}}$

where $R_{q 1}$ and $R_{q 2}$ are the root-mean-square roughnesses of the two non-loaded surfaces. Both groups estimated the film thickness, $h$, either via a regression equation or a smooth film thickness measurement which enabled them to estimate the lambda ratio, $\lambda$, defined as:

$\lambda=\frac{h}{\mathrm{RMS}}$.

They suggested that for a lambda ratio below 1.5, in what could be called the mixed boundary regime, friction depends mainly on what happens at the top of the asperity peaks where a micro-EHD film forms. Evans and Johnson [6] proposed a limiting shear stress rheological model to calculate friction.

Nanbu et al. [7] produced rollers with both random and directional roughness structure and different levels of finish and measured the friction using a two-roller machine. They also measured the film thickness with a capacitance method. From these measurements, they concluded that the observed increase in friction due to roughness was attributable to an increase in lubricant viscosity rather than an increase in shear rate.

All this previous work supports the hypothesis of Jefferis and Johnson [8] that in lubricated rough surface contacts, friction is dominated by the operating conditions at the tops of asperities, where a micro-EHD film forms and the pressure is concentrated. At these asperity peaks, the combination of high pressures and thinner films will have the effect of increasing both the viscosity and the shear rate compared to the valley regions. Thus, the increase in friction that occurs when entrainment speed and thus EHD film thickness are reduced may not arise solely from asperity friction due to solid-solid contact, as is often assumed, but at least in part, from higher shear stress of the micro-EHD film at the asperity conjunctions.

In a later study, Nanbu et al. [9] investigated the influence of the orientation of roughness grooves with the objective of optimizing a traction drive: hence, their goal was to increase friction rather than to decrease it. Their results showed that the surface that gave the highest friction was the one where the grooves were parallel to the entrainment direction (longitudinal roughness). This is in line with a previous experimental study by Johnson and Spence [10]. The conventional explanation for this is that when the grooves are aligned with the entrainment direction, the lubricant can pass through the contact by staying in the valleys in between asperities; hence, the film thickness should be dramatically reduced at the top of the ridges. Various experimental studies on the film thickness $[1,11]$ have shown a clear reduction in the lubricant film thickness at the top of asperities with longitudinal roughness compared to other textures.

Britton et al. [12] studied the effect of roughness on friction in a gear pair. They compared friction of a gear pair where the roughness was oriented transverse to the rolling/ sliding direction to a gear where the surface had been superfinished. They reported a reduction in friction of $20 \%$ in the "smooth" case compared to the rough one confirming that roughness has a detrimental effect on friction.

In an interesting theoretical study, Jacod et al. [13] showed that the friction was greatly influenced by the roughness parameters in the case of parallel ridges. Notably, when the ridges were oriented along the rolling/sliding direction, the resulting friction was much higher than with a smooth surface.

This work presents an experimental study on the influence of roughness on friction in concentrated rolling/sliding contacts. The roughnesses considered are parallel ridges oriented along the rolling/sliding direction, characterized by a certain wavelength and peak-to-valley height. This roughness structure is particularly relevant to rolling bearing applications as a similar roughness geometry may be generated on the bearing raceways of the manufacturing process.

\section{Experimental Techniques and Methods}

\subsection{Ball-on-Disc Rig}

The experiments were carried out on a modified ball-ondisc test rig from PCS instruments (see Fig. 1). In this rig, a 
concentrated contact is produced between a steel ball and a glass disc, which are driven by separate motors that can be controlled independently, enabling studies under rolling/ sliding as well as pure rolling conditions.

The original white light interferometry set-up was modified in order to extend the range of film thickness that can be measured on the rig as well as enable film thickness measurements with rough surfaces at higher speeds. The film thickness measurements were based on imaging optical interferometry (see for example [14]). The modifications included an improved illumination and image acquisition system. For the full description of this experimental set-up, the reader is referred to Guegan et al. [3]. Friction was measured via a torque meter attached to the ball shaft, and the raw signal was recorded in real time. Thus, the film thickness and friction could be measured simultaneously. Relevant temperatures were measured through two RTDs: one monitoring the temperature of the pot and the other the temperature of the lubricant. The accuracy was estimated to be $\pm 0.5^{\circ} \mathrm{C}$.

\subsection{Rough Specimens}

The steel ball specimens with desired roughness structure were produced using a carefully controlled surface finishing process. This technique produces roughness structure parallel to the rolling/sliding direction, and through careful control of tool radius and feed rate, it was possible to obtain roughnesses with a dominant wavelength and peak-

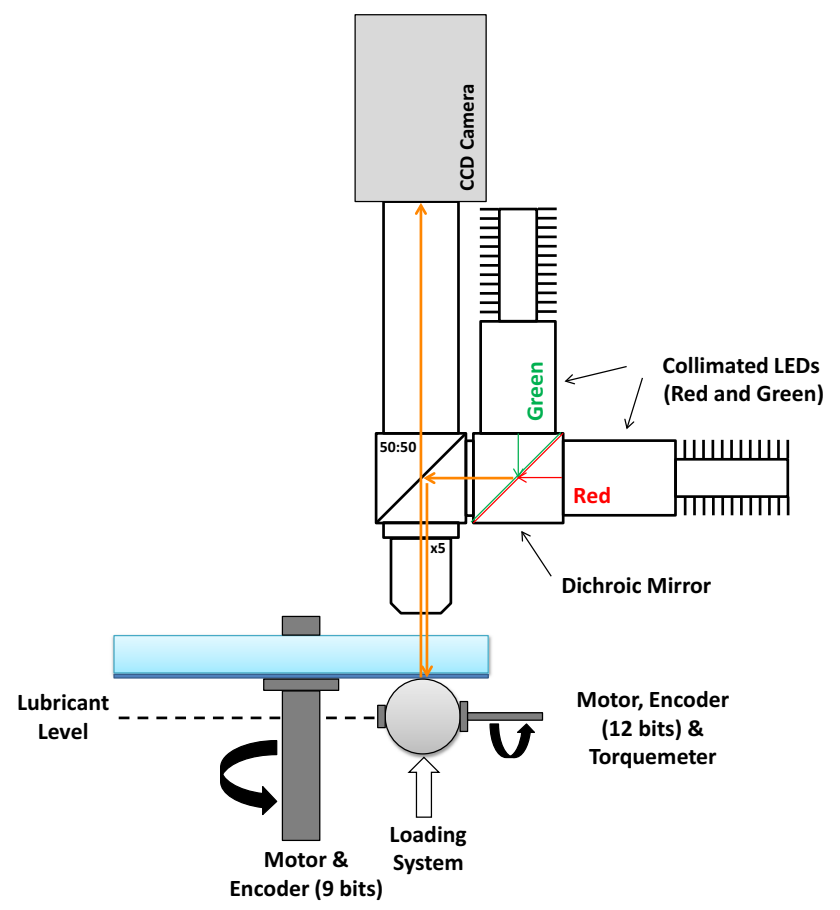

Fig. 1 Ball-on-disc test rig [3] to-valley height. Figure 2 shows an example of surface roughness obtained using this method. Specimens 1 and 2 have very similar peak-to-valley heights but different dominant wavelengths, while specimen 3 has a much higher peak-to-valley height and similar wavelength to that of specimen 1 .

Three roughness structures were tested in this work. They are the same as the ones studied in Guegan et al. [3] in their work on the influence of roughness on film thickness, so that it was possible to correlate the measured friction with previous film thickness results. Table 1 summarizes the dominant characteristics of these surfaces. In addition, for comparison purposes, the standard, "smooth" ball specimens were also used in this study with $R_{q}=20 \mathrm{~nm}$.

In all cases, counterface discs are very smooth $\left(R_{q}=5 \mathrm{~nm}\right)$ and made of glass with a thin chromium and silica layer to enable optical interferometry measurements.

\subsection{Friction Measurements}

At the beginning of each test to measure contact friction a precaution had to be taken to exclude any other sources of friction that may be picked up by the torque meter on the ball shaft due to the intricacies of the rig set-up. The ball shaft is supported by a sealed ball bearing so that when the ball is loaded against the disc seals, the bearing can provide additional resistance, which effectively offsets the contact friction measurement. Some friction will also occur due to spin within the contact since the ball shaft is parallel to the plane of rotation of the disc. To measure this offset, the output of the torque meter was measured in pure rolling with a smooth ball for the range of ball speeds and loads studied and the recorded values used to correct the friction measurements made in rolling/sliding, ensuring that contact friction is measured as accurately as possible.

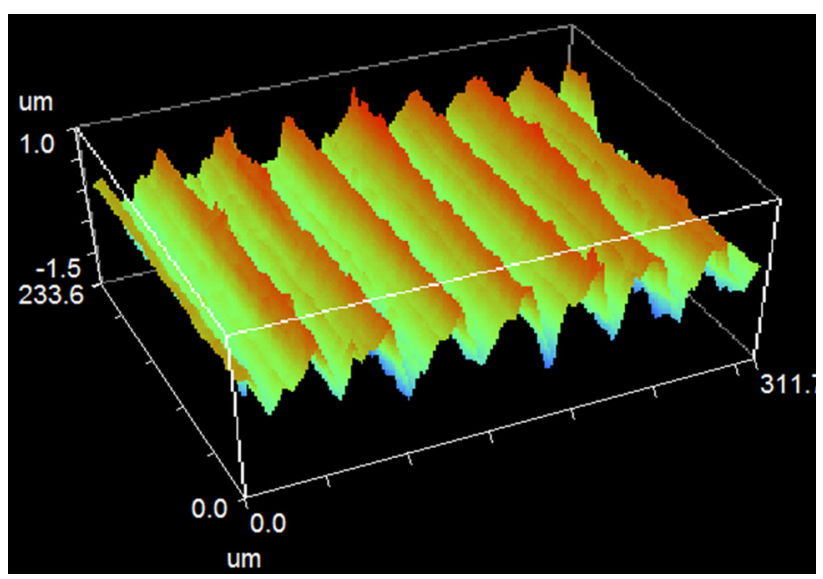

Fig. 2 Example of roughness topography used in the present study 
Table 1 Roughness characteristics of the steel ball specimens tested

\begin{tabular}{llll}
\hline & $\begin{array}{l}\text { Average peak- } \\
\text { to-valley height } \\
(\mu \mathrm{m}): H\end{array}$ & $\begin{array}{l}\text { Dominant } \\
\text { wavelength }(\mu \mathrm{m}) \\
\text { (from FFT analysis) }\end{array}$ & RMS $(\mu \mathrm{m})$ \\
\hline Specimen 1 & 0.52 & 45 & 0.15 \\
Specimen 2 & 0.49 & 19 & 0.15 \\
Specimen 3 & 0.97 & 39 & 0.27 \\
\hline
\end{tabular}

It was important to confirm that the offset did not drift over time and, as evident in Fig. 3, no such evolution was observed. It can be seen that at high speeds, the measurements are highly repeatable, while at lower speeds, below about $100 \mathrm{~mm} / \mathrm{s}$, they are more scattered. This is due to limitations in the speed control of the EHD rig which is not able to control the disc and ball motor speeds to the absolute accuracy needed to achieve pure rolling at these low speeds. Thus, at these speeds, there will always be some amount of sliding (positive or negative). In order to estimate the offset in this low speed range, a best fit was found in the form of a logarithmic parabola.

During each test, the friction was measured using this offset as a reference. Due to the way it was measured, the offset of course includes the rolling component of friction, but this was deemed negligible relative to the contact friction in rolling/sliding conditions.

\subsection{Repeatability of the Friction Measurements}

It was important to ensure the repeatability of the friction measurements on the rough specimens.

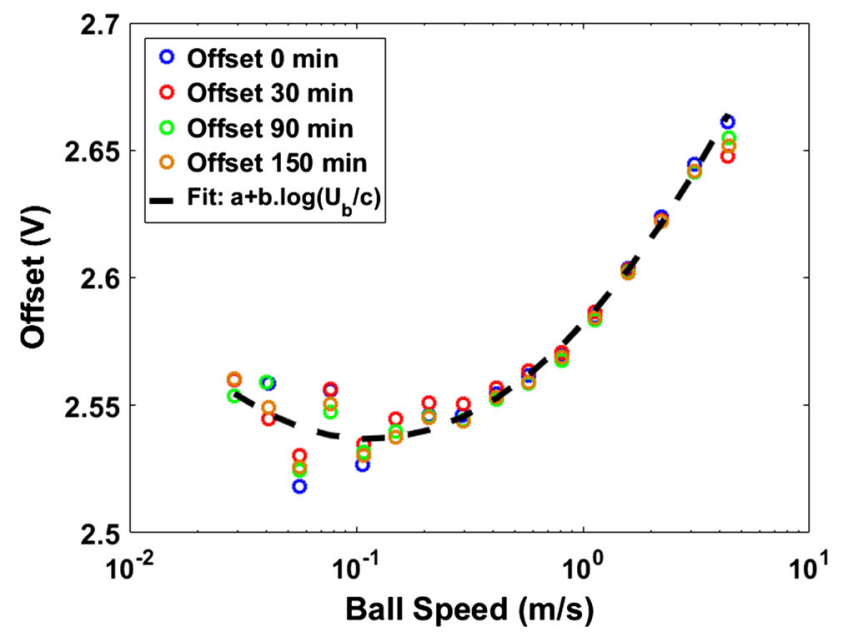

Fig. 3 Raw output of the ball shaft torque meter for a pure rolling contact with smooth specimens (ball $R_{q}=20 \mathrm{~nm}$ and disc $R_{q}=5$ $\mathrm{nm})$ at different times of continuous running at $40{ }^{\circ} \mathrm{C}$. The fitted curve was used as torque meter offset for all measurements of contact friction under rolling/sliding conditions: $U_{\mathrm{b}}=$ ball speed, $a=2.54$, $b=0.009, c=0.11$
In particular, it was necessary to make sure that there was no running-in of the surface during the measurements which would make the results impossible to interpret. Some scratching of the disc's coating was indeed observed after a long period of time which corresponded to the time necessary to measure the film thickness as explained in Guegan et al. [3]. However, the friction measurements only took about 1 min so it was considered that during this short period of time, the roughness of the ball did not vary. Since the scratching on the disc was moderate, we assumed that it would be negligible on the much harder ball.

To ensure the reproducibility of the measurements, the same specimens were tested several times under the same conditions. As shown in Fig. 4 the friction measurements did not vary significantly over time which suggests that the method is repeatable.

\subsection{Experimental Protocol}

Before each test, the steel ball specimens, the glass disc, the ball carriage, the ball shaft and the lubricant pot were cleaned consecutively in toluene and Analar Isopropanol and then dried with a heat gun in order to remove any remaining solvent.

Once cleaned, the different parts were assembled in the lubricant pot which was then filled with oil so that half the ball was submerged. At least $30 \mathrm{~min}$ was required in order for all the parts and the oil temperature to stabilize at the test temperature.

\subsection{Test Conditions}

The specimens were tested in the ball-on-disc rig under the conditions listed in Table 2.

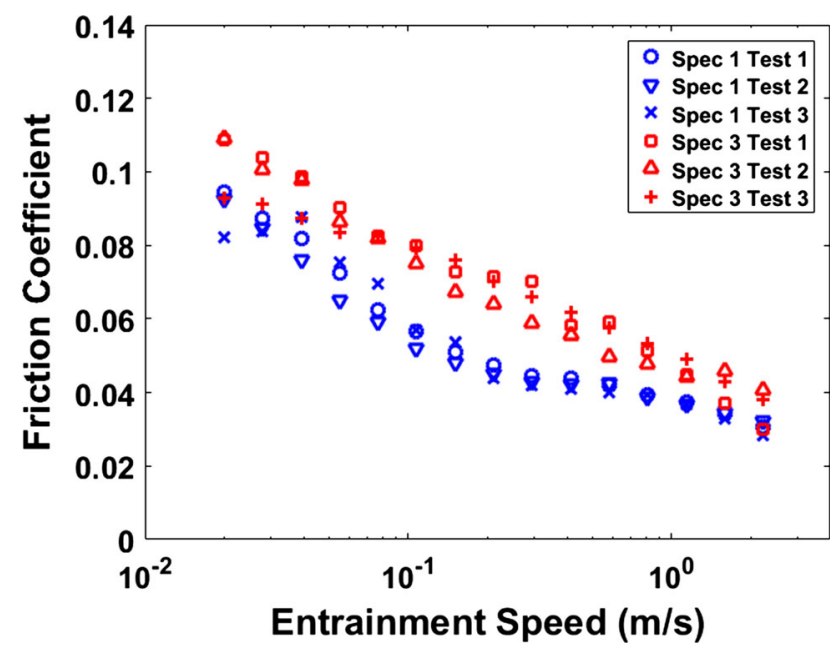

Fig. 4 Examples of Stribeck curves measured for SRR $=50 \%$ with two of the rough specimens 
The SRR was defined as:

$\mathrm{SRR}=\frac{U_{\text {Disc }}-U_{\text {Ball }}}{U_{\mathrm{e}}}$

where $U_{\mathrm{e}}$ is the entrainment speed equal to the average of $U_{\text {Disc }}$ and $U_{\text {Ball }}$.

The load of $20 \mathrm{~N}$ gives a maximum Hertz pressure of $0.527 \mathrm{GPa}$ and a contact diameter of $269 \mu \mathrm{m}$ based on smooth contact predictions for a steel ball on a glass disc. As shown in Guegan et al. [3], for the tested roughnesses the dimension of the contact zone remains similar to the smooth predictions, but the pressure distribution is very different as the load is predominantly borne by the asperities.

Two lubricants with different viscosities were tested. The lower viscosity one was Shell Turbo 68, a Group II base oil with antioxidant additives. The second lubricant was a Group I base oil. The properties of the lubricants were measured and are listed in Table 3. The test temperature was kept constant at $40{ }^{\circ} \mathrm{C}$.

These lubricants and the test temperature were chosen to enable a full span of the lubrication regimes over the entrainment speed range achievable. As can be seen in Fig. 5, at any entrainment speed, the film thickness generated by the more viscous lubricant was approximately twice that of the less viscous one. It should also be noted that the SRR did not have any effect on the measured film thickness: to improve readability, only the pure rolling and $\mathrm{SRR}=100 \%$ cases are shown.

\section{Results}

\subsection{Tests with Smooth Specimens}

The friction was first measured using smooth balls $\left(R_{q}=20 \mathrm{~nm}\right)$. Stribeck curves at various SRRs are displayed in Fig. 6.

The friction was found to increase with SRR over almost all of the entrainment speed range tested $(0.02-2.0 \mathrm{~m} / \mathrm{s}$ equating to $\lambda$ range of $1.3-30)$. In principle, the friction increases continuously with SRR according to the prevailing shear stress versus strain rate relationship.

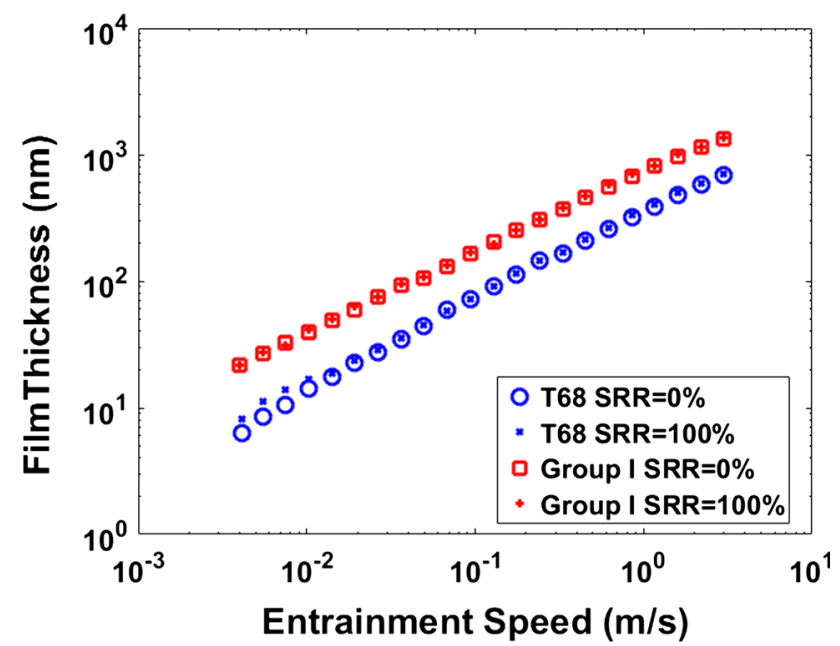

Fig. 5 Film thickness versus entrainment speed for the two test lubricants with smooth specimens at $40{ }^{\circ} \mathrm{C}$ in pure rolling and for $\mathrm{SRR}=100 \%$

However, in practice, at high sliding speed the friction levels out at a maximum and then starts to decrease due to heating of the lubricant film. Rolling bearings generally operate at low SRRs, well below this maximum. In the current set-up, most of the friction measurements are below the maximum because of the relatively low contact pressure which results in low shear stress and thus low temperature rise. A notable exception can be seen in the T68 curves at the highest entrainment speeds and SRRs (and thus at the highest sliding speeds), where the Stribeck curve at $\mathrm{SRR}=100 \%$ falls below the one at $\mathrm{SRR}=50 \%$. Another interesting feature apparent in Fig. 6 is that, for a given oil, all the friction curves seem to converge at high speeds.

For all SRRs and oils, friction is relatively high at low speeds and decreases with speed. Apart from the Stribeck curve for $\mathrm{T} 68$ at $\mathrm{SRR}=100 \%$, all curves reach the first minimum at relatively low speeds before the friction starts going up again. The classical explanation is that at low film thickness, the contact operates in the mixed lubrication regime where friction results from a combination of fluid friction and boundary friction. When full separation occurs, the friction is dominated by the fluid friction and starts to increase again in accord with the shear stress versus strain
Table 2 Experimental conditions

Table 3 Oil properties

\begin{tabular}{llll}
\hline Temperature & Load & Speed range & SRR \\
\hline $40.0 \pm 0.5^{\circ} \mathrm{C}$ & $20 \mathrm{~N}$ & $0.02-2 \mathrm{~m} / \mathrm{s}$ & 50 and $100 \%$ (a few additional tests were done at lower SRRs)
\end{tabular}

\begin{tabular}{lccl}
\hline & Viscosity at $40{ }^{\circ} \mathrm{C}(\mathrm{cP})$ & Viscosity at $100{ }^{\circ} \mathrm{C}(\mathrm{cP})$ & Refractive index at $40{ }^{\circ} \mathrm{C}$ \\
\hline Shell Turbo 68 & 57.7 & 7.1 & 1.469 \\
Group I & 233.7 & 24.3 & 1.470 \\
\hline
\end{tabular}



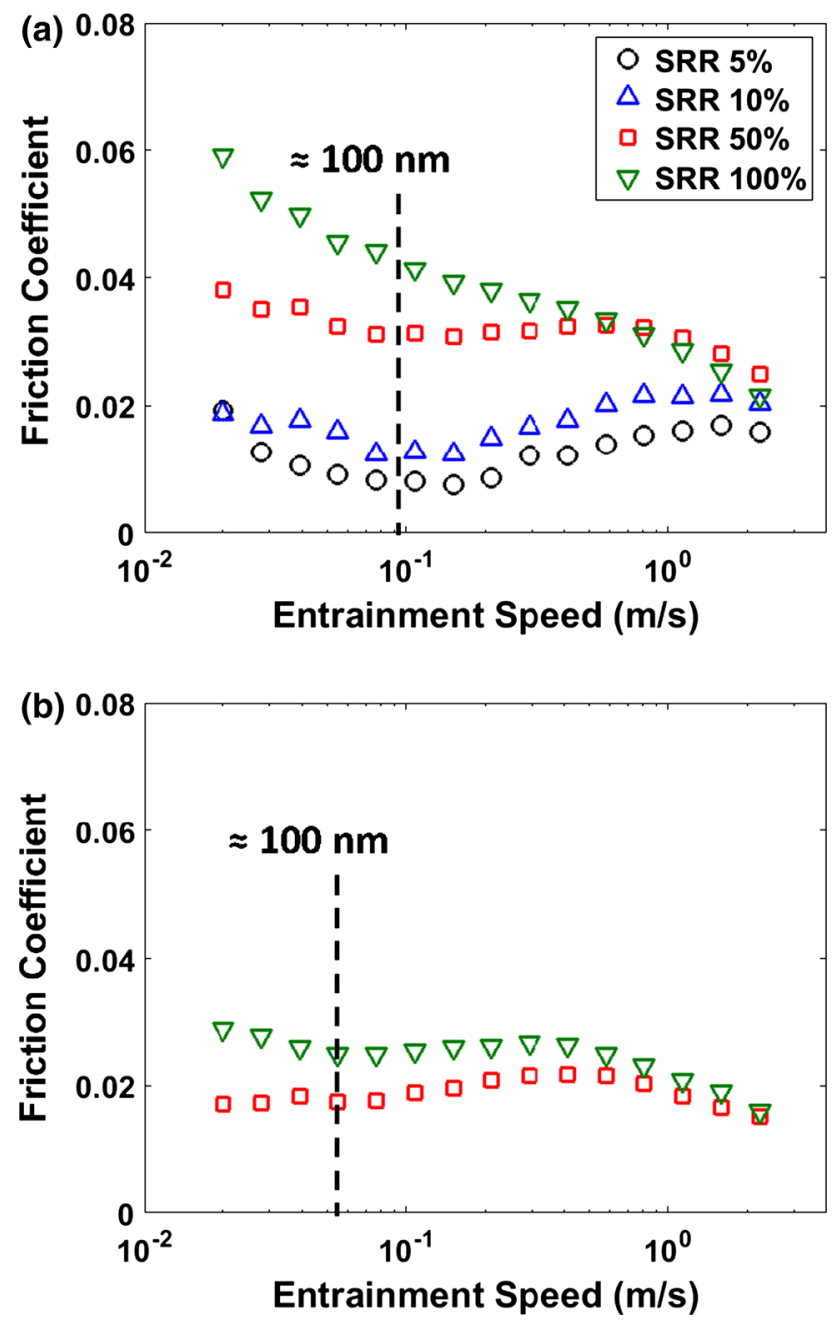

Fig. 6 Stribeck curves measured with smooth ball specimens $\left(R_{q}=20 \mathrm{~nm}\right)$ at various SRRs; a T68 oil and b the Group I oil. The oil film thickness at which the transition from mixed to full EHD lubrication occurs is also shown $\left(P_{\text {mean }}=3.5 \times 10^{8} \mathrm{~Pa}, T=40^{\circ} \mathrm{C}\right)$

rate relationship, so that this minimum is usually considered to be the transition from mixed lubrication to full-film lubrication. For both oils, this indicates that the transition corresponds to a lambda ratio $\lambda \approx 5$.

\subsection{Tests with Rough Specimens}

Friction measurements were taken on rough balls under the same operating conditions as used with the smooth specimens.

The Stribeck curves measured in the case of a smooth and the three rough specimens for T68 and the group I oil are shown, respectively, in Figs. $7 \mathrm{a}, \mathrm{b}$ and $8 \mathrm{a}$, b. It can be noted that at low speeds, the rough specimens systematically generate a higher friction compared to the smooth specimen. In general, as the speed increases and the film builds up, the rough specimen friction starts to decrease
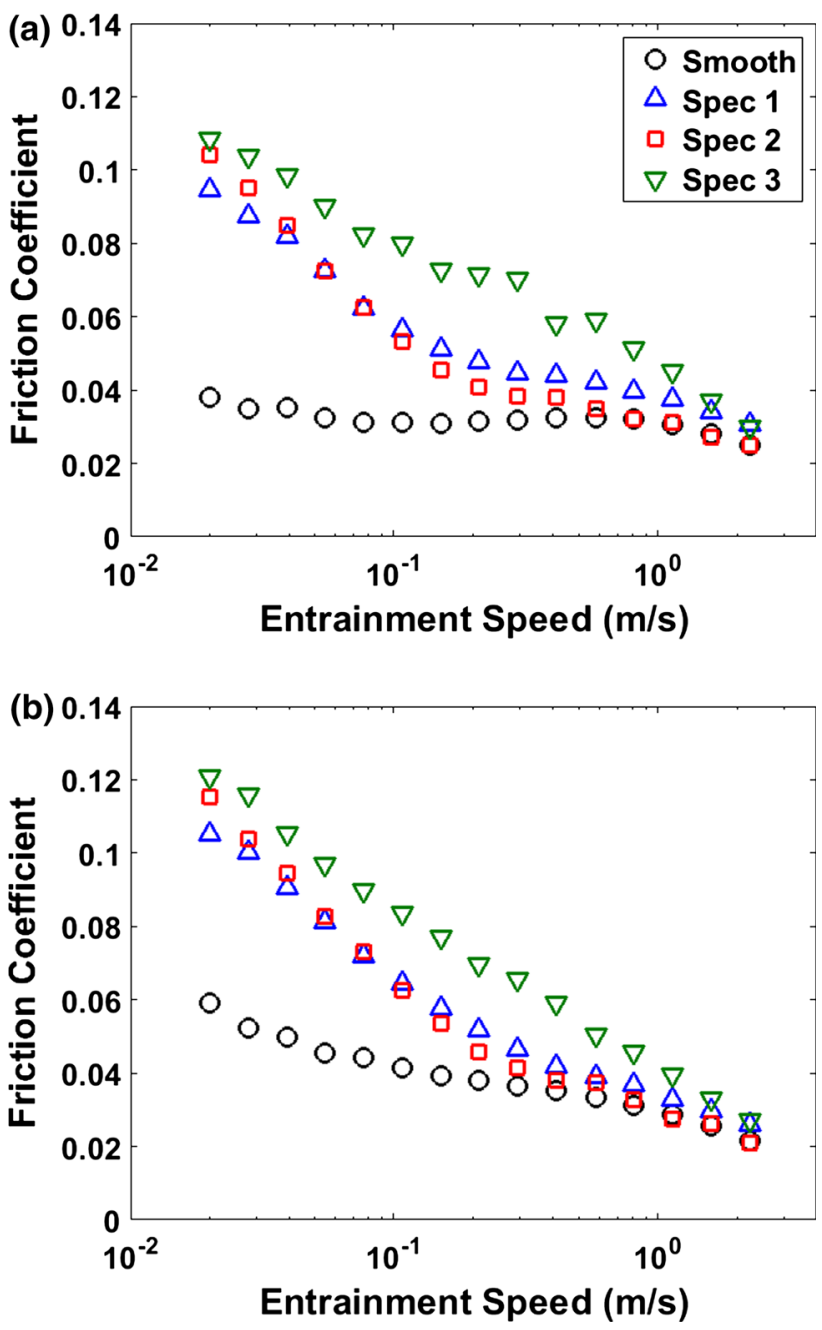

Fig. 7 Comparison of the Stribeck curves for smooth and rough specimens, with T68 oil. a SRR $=50 \%$ and $\mathbf{b}$ SRR $=100 \%$

down to the point where it coincides with the friction of the smooth specimen. This may intuitively be expected since at higher speeds, the oil film grows to provide full separation between the surfaces rendering roughness effects on friction negligible. In one measurement (specimen 2 with the Group I base oil), a slight difference in friction remains at high speed. It is believed that this comes either from experimental error or some drift in the torque meter offset.

From Figs. 7 and 8, it is apparent that specimens 1 and 2 , which have different wavelengths but the same peak-tovalley height, produce very similar friction under all conditions. Furthermore, the friction measured with specimen 3 , which has the highest peak-to-valley height, was significantly higher than with the other specimens. These results suggest that the roughness peak-to-valley height has much more influence on contact friction than the wavelength.

As expected, a higher SRR gives a higher friction. 

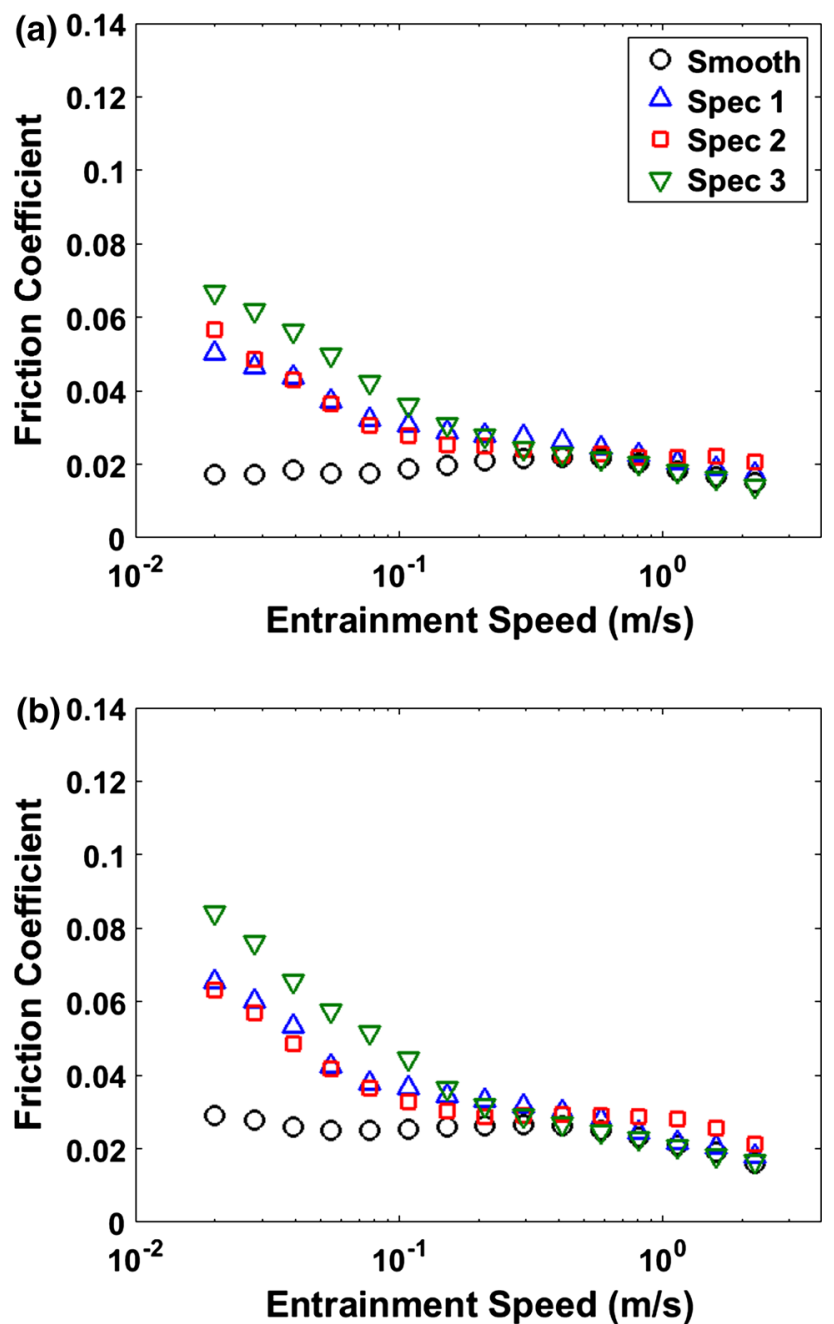

Fig. 8 Comparison of the Stribeck curves for smooth and rough specimens, with the Group I oil a SRR $=50 \%$ and b SRR $=100 \%$

The results obtained with the two oils follow similar friction trends. The absolute values of friction coefficient are of course different, as may be expected, as is the transition speed at which the rough surface friction becomes coincidental with the smooth surface friction. This latter observation can be explained by the fact that the more viscous Group I base oil generates a thicker fluid film for a given speed, so that the transition to full-film EHD lubrication occurs earlier.

In order to quantify the increase in friction due to specific roughness, the difference in friction coefficient between the smooth and rough contact for given lubricant, contact conditions and roughness properties is plotted in Fig. 9.

It can be seen that the increase in friction due to the specific roughness is almost independent of the SRR for the conditions tested, i.e. for positive SRR below $100 \%$. Hence, a distinction between "smooth" and "rough" surface friction can be made in a manner different from the
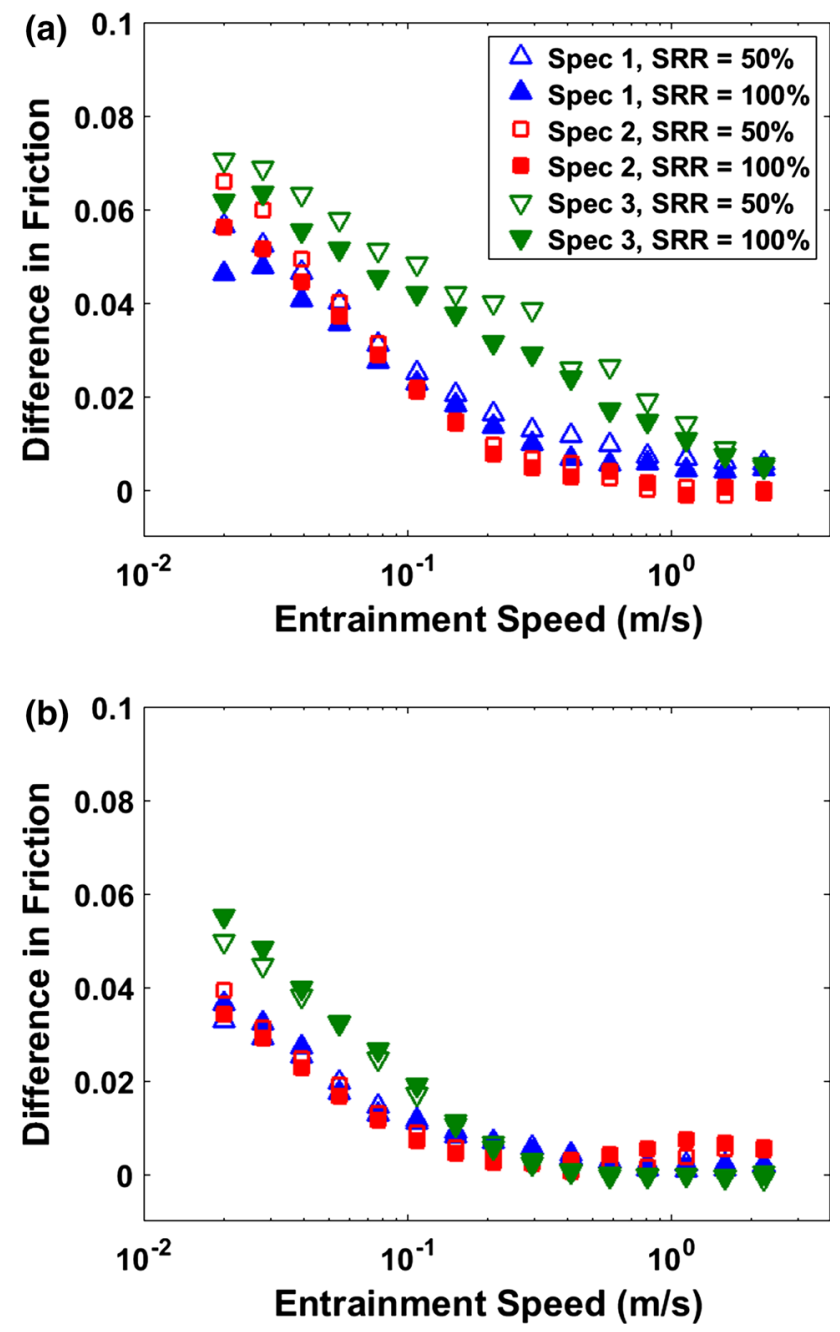

Fig. 9 Difference in friction coefficient between the smooth case and the rough case at given contact conditions with a T68 and b the Group I base oil

usual separation into "fluid" and "boundary" friction by accounting for the effects of roughness on pressure distribution and shear rate (see for example [15]).

\section{Discussion}

\subsection{Smooth Contact Friction Behaviour}

The results in the smooth contact case are in line with results from previous authors $[4,6]$. In principle, above $\lambda \approx 3$, there should only be minimal contact, so the transition to full film might be expected there. A hypothesis made by some other authors [8] is that, even though there is full separation, the traction remains dominated by the local shear rate at the top of asperities where a micro-EHD film forms and the pressure is high. If the pressure is localized, the local viscosity of the lubricant will be much higher than 
that at the average or Hertz pressure. In addition, the film thickness at the top of asperities will be thinner than the nominal film thickness so the shear rate will be higher. These two phenomena may contribute to increase the friction in this regime even if there is no actual solid-tosolid contact.

Above $\lambda>5$, it is evident in all cases tested that the friction does not increase indefinitely with speed; at the highest speeds, it starts decreasing again. Lafountain et al. [4] explain this by the fact that at high shear rate, the lubricant starts to heat up, which decreases the viscosity and causes a drop in friction. To prove their hypothesis, they estimated the temperature rise in the contact and used it to obtain thermally corrected friction curves.

An alternative way of plotting the Stribeck curves, also due to Lafountain et al. [4], involves calculating the mean shear rate, from the ratio of the sliding speed and the central film thickness.

Alongside the friction measurement, a theoretical friction curve using an Eyring rheology model has been plotted in Fig. 10 (with $\alpha=19.2 \mathrm{GPa}^{-1}$ and $\tau_{\mathrm{E}}=4.7 \mathrm{MPa}$ ). It can be seen that for the low SRRs and full-film conditions, the theoretical curve describes the evolution of friction quite well. At higher SRRs and shear rates, this curve does not seem to model the friction so well. This difference between experience and theory is often attributed to thermal effects. To test this hypothesis, the temperature rise in the contact was estimated using Eq. (4) taken from Spikes and Jie [16] which estimates the in-contact oil film temperature rise as the sum of the mean flash temperature rise, $\Delta \bar{T}_{\text {surf }}$, which corresponds to the transient rise in temperature of the surfaces as they cross the contact, and the mean oil temperature rise above the surface temperature due to shearing, $\Delta \bar{T}_{\text {oil }}$ :

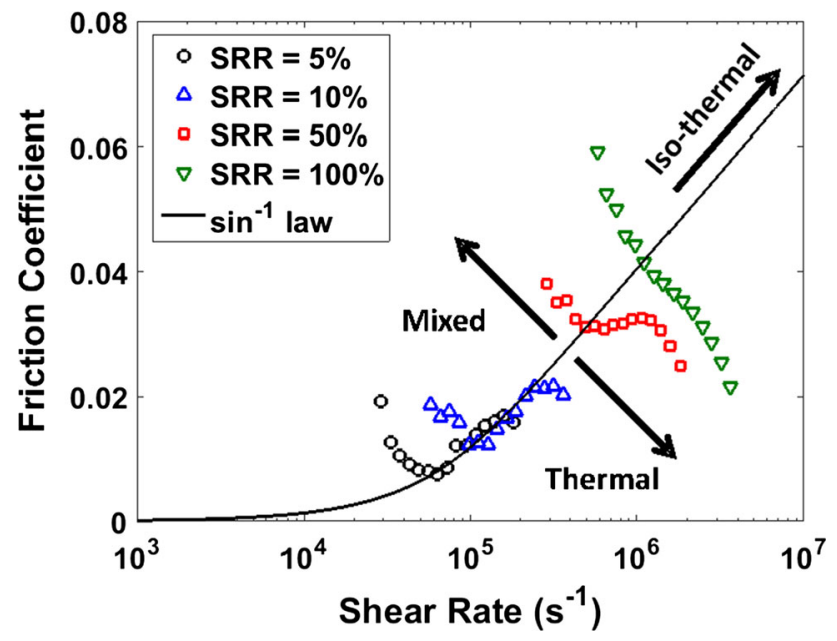

Fig. 10 Friction against shear rate at different SRRs with T68; the different lubrication regimes are shown along with a $\sinh ^{-1}$ curve calculated with $\alpha=19.2 \mathrm{GPa}^{-1}$ and $\tau_{\mathrm{E}}=4.7 \mathrm{MPa}$

$$
\begin{aligned}
\Delta \bar{T} & =\Delta \bar{T}_{\text {surf }}+\Delta \bar{T}_{\text {oil }} \\
& =\frac{1}{(2 \pi K \rho c)^{0.5}}\left(\frac{2 a}{U}\right)^{0.5} \bar{\tau} \Delta U+\frac{h}{8 K_{\text {oil }}} \bar{\tau} \Delta U
\end{aligned}
$$

where $K$ and $K_{\text {oil }}$ are the respective thermal conductivities of the surface material and the oil, $\rho$ the density of the material, $c$ its specific heat capacity, $a$ the half width of the contact, $U$ the entrainment speed, $\Delta U$ the sliding speed, $\bar{\tau}$ the mean shear stress over the contact and $h$ the central film thickness. This equation considers that the two contacting materials are the same; however, in this study, the ball was made of steel and the disc of glass. To calculate the flash temperature rise, the flash temperature was calculated twice, assuming steel/steel and glass/glass contacts. Then, according to Archard [17], the temperature rise can be calculated via:

$\frac{1}{T_{\text {total }}}=\frac{1}{T_{\text {steel }}}+\frac{1}{T_{\text {glass }}}$.

Under the conditions tested and at SRR $=5 \%$, the temperature rise was found to be negligible, which is consistent with the fact that the friction curve at this SRR follows the Eyring model [Eq. (1)]. At higher SRRs, the temperature rise was found to be significant, reaching $14{ }^{\circ} \mathrm{C}$ at the highest SRR of $100 \%$.

As shown in Fig. 11, the deviation of the measured friction from the Eyring model evolves linearly with increasing temperature with the gradient of this straight line being $-0.83 \mathrm{MPa} /{ }^{\circ} \mathrm{C}$. This linear evolution along with the value of the slope is consistent with Spikes and Zhang [18]. Using this gradient and the calculated temperature rise, the measured friction data have been thermally corrected. As shown in Fig. 12, the corrected curves seem to fall very close to the Eyring model, which supports

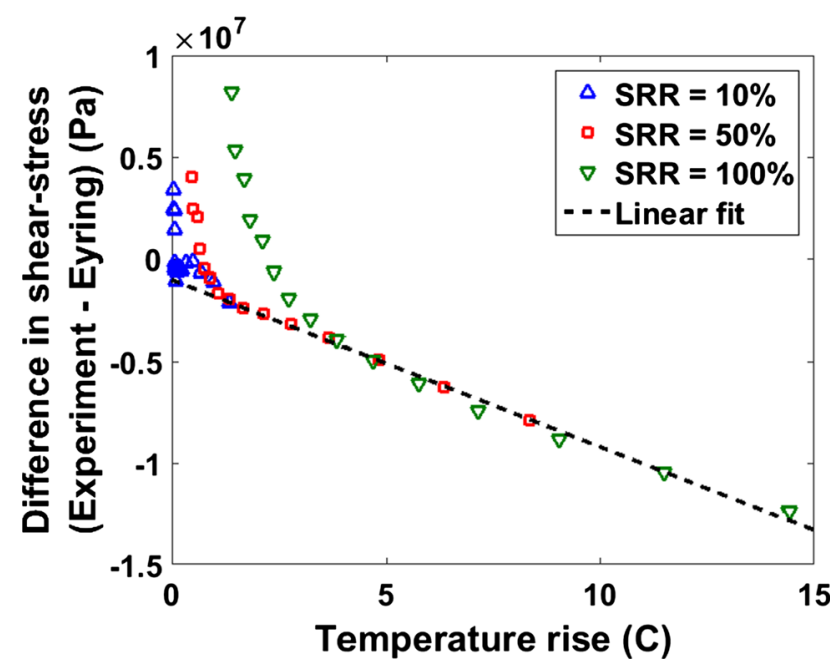

Fig. 11 Difference between measured shear stress and Eyring model versus calculated temperature rise 


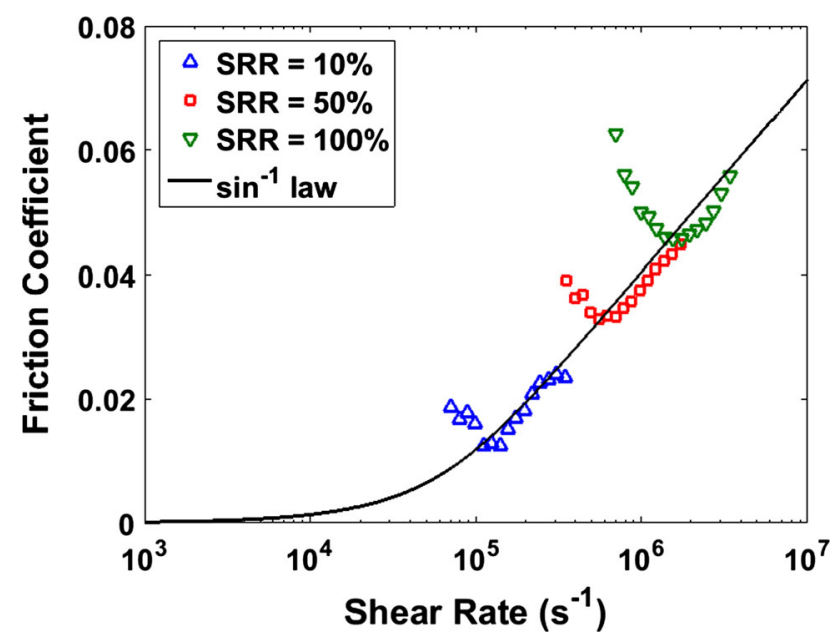

Fig. 12 Thermally corrected friction curves versus shear rate; the $\sinh ^{-1}$ law is also plotted

the thermal origin of the drop in friction at high SRRs and shear rates.

To summarize, most of the smooth case curves show similar behaviour regardless of the operating conditions:

- When $\lambda<5$, the contact operates in mixed lubrication, which does not mean that there is necessarily solid-tosolid contact since even if the contacting surfaces are fully separated, the asperities may still influence the pressure and the shear rate as seen by Guegan et al. [3]. The rheological behaviour of the micro-EHD film that forms at the asperities may have a decisive influence on the friction.

- For $\lambda>5$, two behaviours can be observed. Either the friction follows a trend well described by a $\sinh ^{-1}$ relationship between shear rate and shear stress (for example) and increases with speed, or the friction decreases with speed. The latter occurs at high sliding speeds and is due to shear heating.

The results for T68 at SRR $=100 \%$ suggest that if the sliding speed is very high, it is possible to observe conditions completely dominated by thermal effects.

\subsection{Influence of SRR on Rough Surface Friction}

Like in the smooth case, for rough surfaces a higher SRR results in a higher friction. However, as shown in Fig. 9, the extra contribution of roughness to the overall friction (i.e. subtracting the friction for the smooth case under the same conditions) is largely independent of the slide-roll ratio. If $F_{50}, F_{100}, S_{50}$ and $S_{100}$ are the friction coefficients produced at a SRR $=50 \%$ and a SRR $=100 \%$ for, respectively, a rough and a smooth specimen at the same speed and load, then the following relationship holds:

$$
\begin{aligned}
& F_{50}-S_{50}=F_{100}-S_{100} \\
& \quad \Rightarrow S_{100}-S_{50}=F_{100}-F_{50} .
\end{aligned}
$$

This means that the increase in friction due to a higher SRR will be the same whether the surface is rough or not. Hence, the friction can be split in a "smooth" component that varies with the SRR and a rough component that is independent of it. In order to validate this observation, additional tests were carried out with specimen 1 at $\mathrm{SRR}=5$ and $10 \%$. These are included in Fig. 13. It can be seen that over this wide range of SRRs, the difference in friction between the rough and smooth cases remains predominantly dependent on the entrainment speed and the level of sliding has a minimal effect.

The independence of the rough component of friction from the SRR may be obvious if this rough friction component is assumed to originate from the solid-to-solid contact, in which case the local friction coefficient will be a sliding speed-independent boundary friction coefficient. However, given the high pressures at the asperity peaks, another explanation could be that in the rough contact, the fluid reaches a limiting shear stress locally at asperity conjunctions, which would also make the roughness contribution to overall friction independent of SRR.

It was shown by Guegan et al. [3] that with rough specimens, the film thickness varies around a central line that is well represented by the smooth case at the same entrainment speed. This difference in film thickness distribution gives rise to a shear rate distribution which has a maximum at the asperity peaks where the film thickness is the thinnest and a minimum in the valleys where the film is the thickest. Similarly, the pressure was found to fluctuate around the smooth "Hertzian" case, with high pressures at the top of the ridges and much lower pressures in the

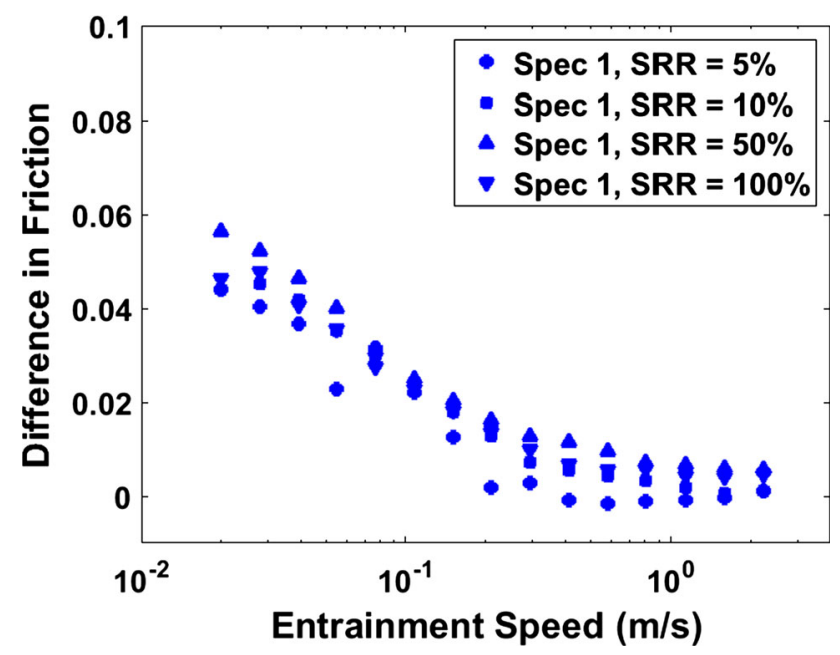

Fig. 13 Influence of the SRR on the difference in friction between the specimen 1 and a smooth specimen 
valleys. Present results show that the smooth component of friction seems to be affected by the average value of the shear rate and the pressure distribution rather than the local variations. In particular, it was shown in the previous section that thermal effects need to be taken into account in order to explain the evolution of the smooth component of friction. Given that the increase in friction due to roughness was shown to be independent of the SRR, it can be deduced that thermal effects have similar influence on friction in both smooth and rough lubricated contacts. This agrees with the analysis by Johnson and Greenwood [19] who showed that for smooth surface contact, a mean temperature approach can be used to estimate the temperature rise in an EHD contact.

\subsection{Influence of Roughness Properties on Measured Friction}

For the tested roughness structures with ridges parallel to rolling direction, the roughness wavelength was not found to have a significant influence on the measured friction. Guegan et al. [3] also observed that for the same roughnesses, the wavelength had no influence on the film thickness.

To assess the effects of the peak-to-valley height or RMS roughness, an attempt was made to relate the measured friction coefficients to lambda ratio for all tested conditions. This was possible since the EHD film thickness was measured for all the surfaces under the same test conditions in a related previous study (see [3]). The lambda ratio for rough contacts is now defined as:

$\lambda=\frac{h_{\mathrm{AVG}}}{\mathrm{RMS}}$

where $h_{\mathrm{AVG}}$ is the measured average film thickness in the contact and RMS is the composite roughness of the contacting surfaces. Given that the disc is very smooth (RMS $\approx 5 \mathrm{~nm}$ ), the composite RMS is dominated by the RMS roughness of the ball. The values used for the RMS of the rough specimens are the ones listed in Table 1, where specimens 1 and 2 have an RMS of $150 \mathrm{~nm}$, specimen 3 has an RMS of $270 \mathrm{~nm}$, and the smooth specimen has an RMS roughness of $20 \mathrm{~nm}$.

The Stribeck curves previously measured are plotted as friction coefficient versus lambda ratio in Fig. 14a, b for T68 and Fig. 15a, b for the Group I oil.

In the plots of Figs. 14 and 15, friction curves measured with smooth and different rough specimens all fall on the same general curve for the given oil and SRR. The only exception is Fig. 14b (Group I oil, SRR $=100 \%$ ) where the friction measured with the smooth specimen does not lie on the same curve as that measured with the rough specimens. The smooth case lambda ratios were calculated
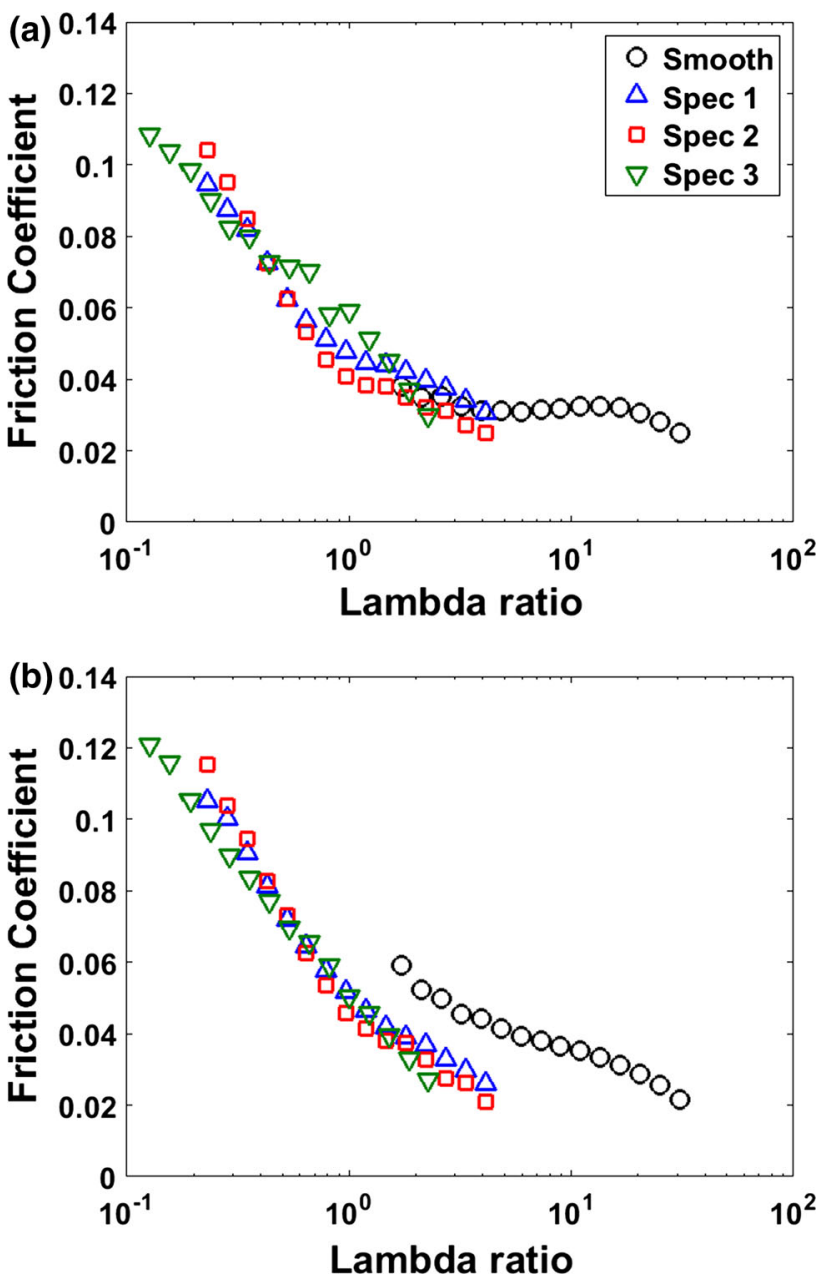

Fig. 14 Measured friction against lambda ratio with T68 oil a SRR $=50 \%$ and $\mathbf{b}$ SRR $=100 \%$

with the composite RMS roughness of $20 \mathrm{~nm}$ (ball $R_{q}=20 \mathrm{~nm}$, disc $R_{q}=5 \mathrm{~nm}$ ). However, given the use of rough ball specimens, it is likely that some scratches formed on the disc coating during the tests so that the actual composite RMS was probably higher. Indeed, if the composite RMS for this smooth case was only slightly higher at $30 \mathrm{~nm}$ (equivalent to disc RMS $\sim 20 \mathrm{~nm}$ ), the smooth case friction curve would coincide with all other Stribeck curves for rough cases. As the composite RMS roughness is dominated by the roughness of the rougher surface, the lambda ratio in the rough cases would not be affected by such scratches and moderate increase in the disc roughness.

It is clear from the presented results that for a given set of conditions, the roughest specimen (highest peak-tovalley height) generates a higher level of friction than the other specimens. Trends apparent in Figs. 14 and 15 suggest that this increase in friction can be solely described by the associated decrease in lambda ratio. This is further supported by Fig. 16, which plots the difference in friction 

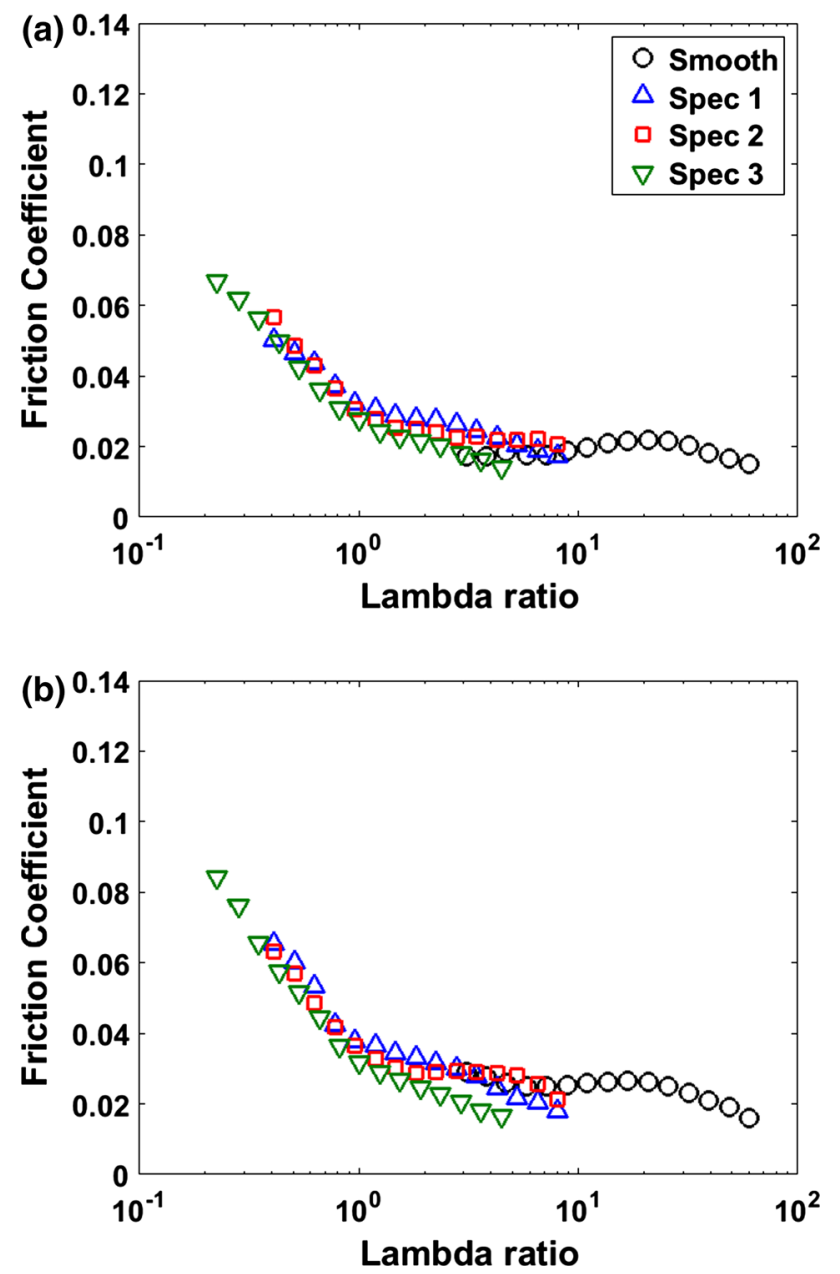

Fig. 15 Measured friction against lambda ratio with the Group I oil a $\mathrm{SRR}=50 \%$ and $\mathbf{b} \mathrm{SRR}=100 \%$

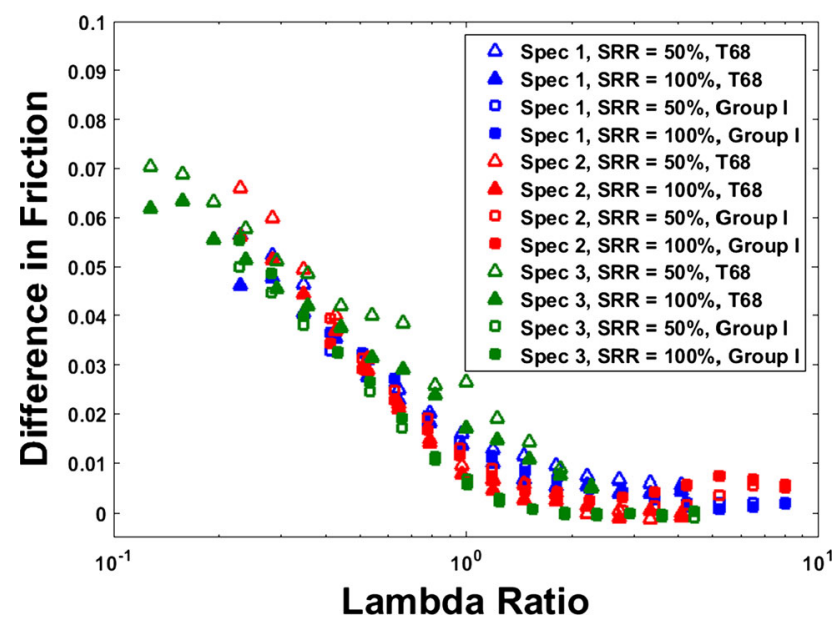

Fig. 16 Difference in friction versus lambda ratio for the three specimens, the two SRRs and the two oils tested between the rough and smooth cases against the lambda ratio for all specimens, SRRs and oils tested.

It can be seen that all results appear to belong to the same master curve, i.e. the contribution of roughness to the total friction is well described by the lambda ratio. Furthermore, this master curve shows that the transition from mixed lubrication to full-film EHD lubrication occurs at a lambda ratio that is between 2 and 5, which is in accordance with the most previous studies $[5,6]$. It implies that the two oils have similar boundary friction coefficients.

\subsection{Potential Sources of Friction in Lubricated Rough Contacts Under Mixed Lubrication Conditions}

In order to interpret the influence of roughness on friction in more detail, it is interesting to examine the point at which rough contact friction stops being higher than the friction in the smooth case for the same SRR and entrainment speeds, i.e. the difference in friction shown in Fig. 9 tends to 0 (or levels out within experimental error). As the film thickness was measured at the same time as friction, it was possible to establish directly whether there remained any asperity contact or not at this transition point.

The evolution of the difference in rough and smooth friction with entrainment speed is plotted alongside the measured minimum film thickness in Figs. 18 and 19, for T68 and the Group I oil, respectively. It should be noted that there is no single minimum film value in rough contact unlike in the smooth case. The film thickness measurements in rough contacts used in this discussion are fully explained in Guegan et al. [3], but essentially the minimum

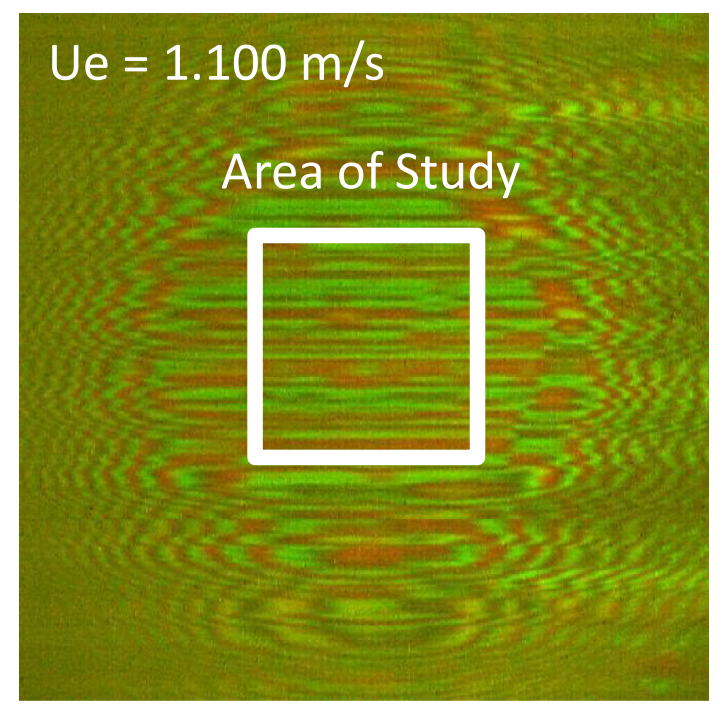

Fig. 17 Interferogram of a rough specimen used to obtain film thickness maps; the region considered for the statistical analysis is also shown (from [3]) 

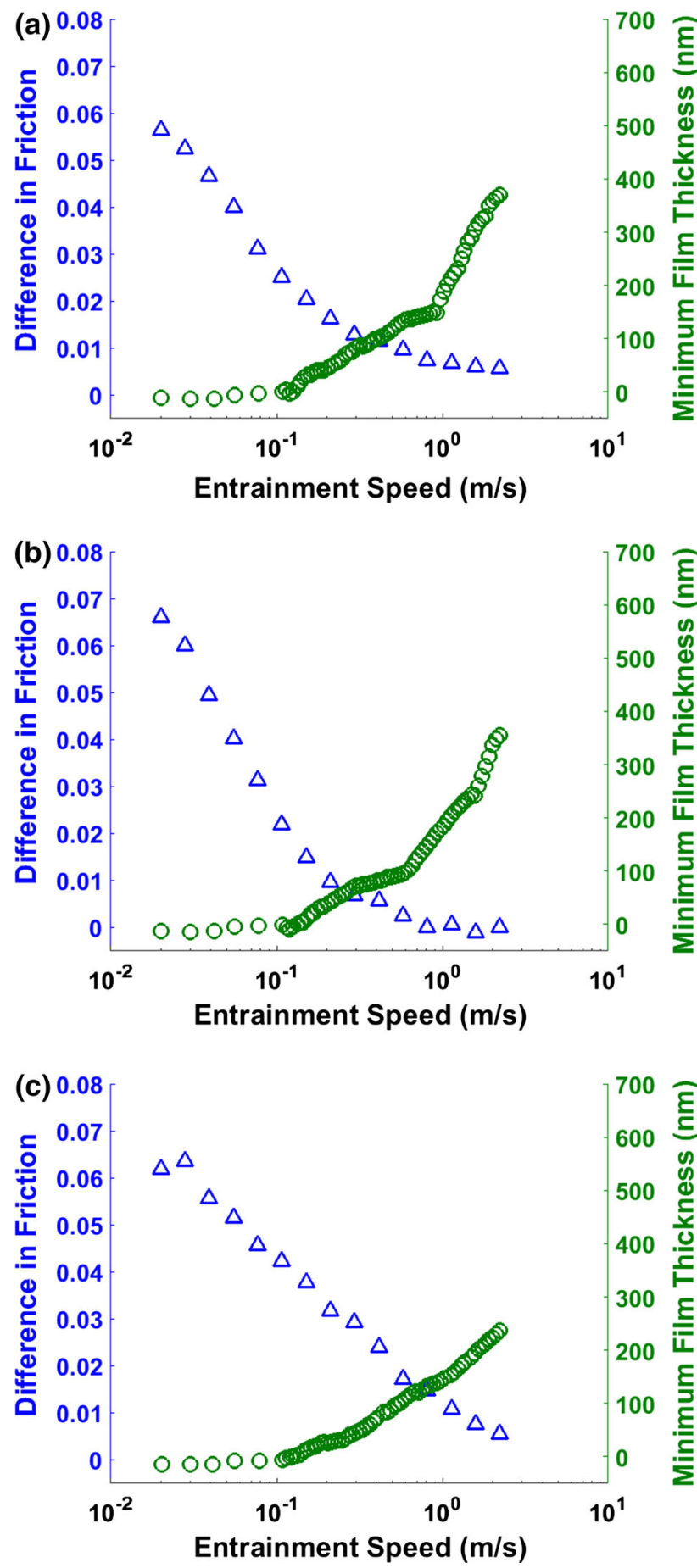

Fig. 18 Evolution of the difference in friction between rough and smooth cases and the corresponding minimum film thickness plotted against entrainment speed; T68 oil a specimen 1, b specimen 2 and c specimen 3

film value is taken to be the average of the lowest $1 \%$ of points in the region defined by the square shown in Fig. 17.

As expected, the evolution of the two measurements have opposite trends: the difference in friction is high at low speed and decreases as the lubricant film builds up, while the minimum film starts flat and increases with speed.

One conventional description of the mixed lubrication regime consists in separating the friction into a fluid component and a boundary one. The results shown here somewhat contradict this assumption. In every case, after lift-off the roughness continues to have some influence on the friction, and it is only when the lubricant film of 200-300 nm separates all of the contacting surface asperities that this influence vanishes completely.

Although it is counter-intuitive, this effect could be expected when considered in parallel with the pressure results of Guegan et al. [3]. It was shown that as the lubricant film builds up, the pressure is redistributed towards the asperity peaks forming a micro-EHD film. This peak of pressure at the asperities, even in the case of a full lubricant film, may explain the delay between the full separation of the contacting surfaces and the convergence between rough and smooth friction. The fact that it does not depend on the SRR, as seen in Sect. 4.2, suggests that the increase in friction is not only due to solid-to-solid contact, but also that the fluid must be reaching a limiting shear stress. However, as seen in Fig. 20, the surface lift-off does seem to affect the rate at which the friction converges towards the smooth friction. This transition zone seems to occur for $\lambda=0.8-3$. This upper limit of 3 is relatively close to the value of 5 found for the smooth specimen. The difference could be related to the difference between the geometries of the roughness of the smooth specimens (random) and that of the rough specimens (parallel ridges).

Another possible explanation for this delay in convergence of smooth and rough friction may be the difference in which minimum film and friction are averaged over the contact: the minimum film thickness is measured only in the central square of the contact, while the friction is averaged over the whole contact. It is possible that greater asperity contact occurs in the region outside the central square where film is measured, giving higher average friction than expected for the measured film thickness at any given point.

\subsection{Prediction of Friction in Lubricated Rough Contacts}

The full prediction of the friction based on the roughness parameters and the oil properties does not seem possible within the current state of the discipline as this would require not only a very accurate knowledge of the local conditions of pressure and shear rate within the contact, but also a correct model for the fluid rheology. That being said, there have been some formulae proposed to calculate the friction in the mixed regime, knowing the lambda ratio, the boundary friction and the fluid friction. In particular, Olver and Spikes [20] suggested the equation: 

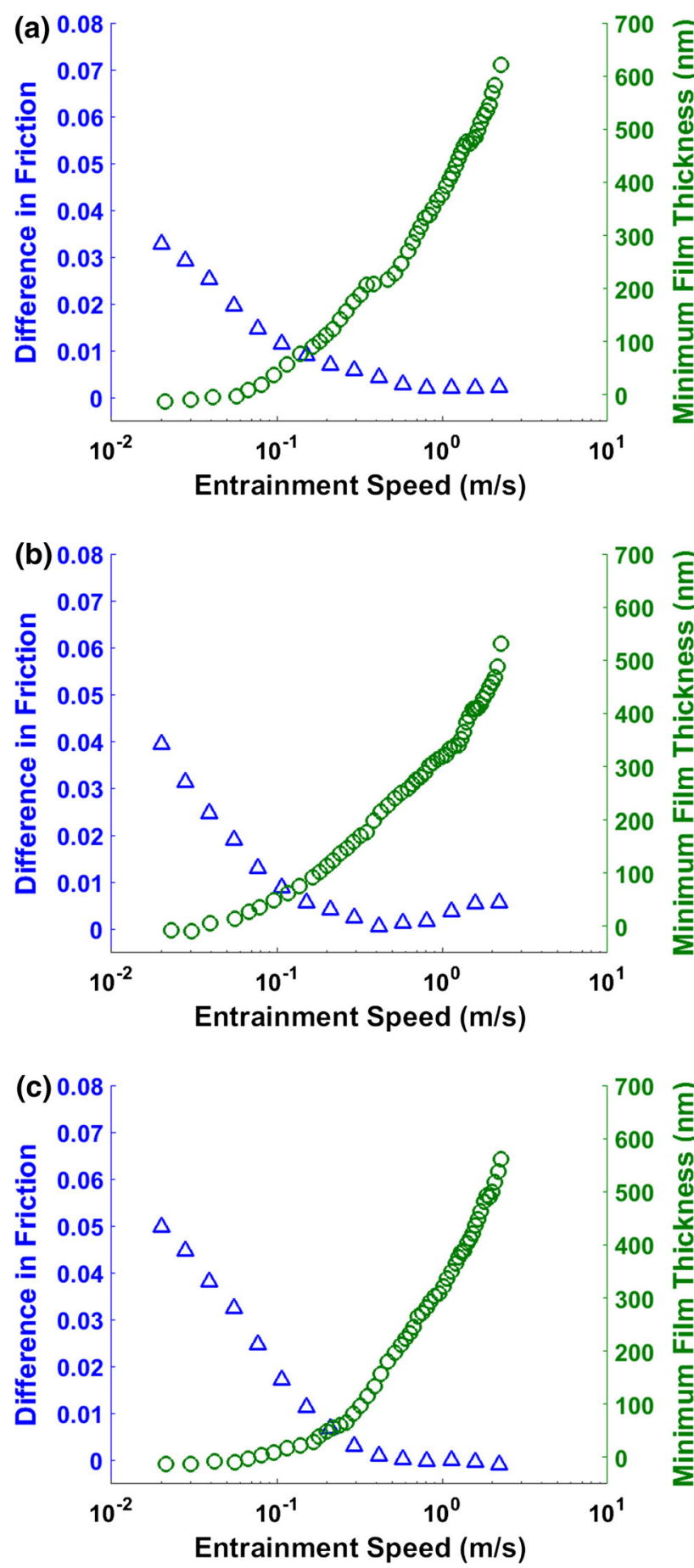

Fig. 19 Evolution of the difference in friction between rough and smooth cases and the corresponding minimum film thickness plotted against entrainment speed; Group I oil a specimen 1, b specimen 2 and $\mathbf{c}$ specimen 3

$\mu=\mu_{\mathrm{f}}+\frac{\mu_{\mathrm{b}}-\mu_{\mathrm{f}}}{(1+\lambda)^{m}}$

where $\mu$ is the coefficient of friction, $\mu_{\mathrm{f}}$ the fluid friction, $\mu_{\mathrm{b}}$ the boundary friction, $\lambda$ the lambda ratio and $m$ a constant, though they do not explain the derivation of this equation. According to the authors, $m$ should be around two. In our experiments, we found that the best results were obtained for $m$ between 2 and 3 and $\mu_{\mathrm{b}}=0.14$ which is a reasonable value for the boundary friction for a steel on glass contact.

Figure 21 compares measured friction with the predictions obtained using Eq. (8). It can be seen that the fits are quite good, especially as they seem to predict non-trivial characteristics of the curves, such as the concavity of the higher RMS Stribeck curve and the convexity of the lower RMS ones.

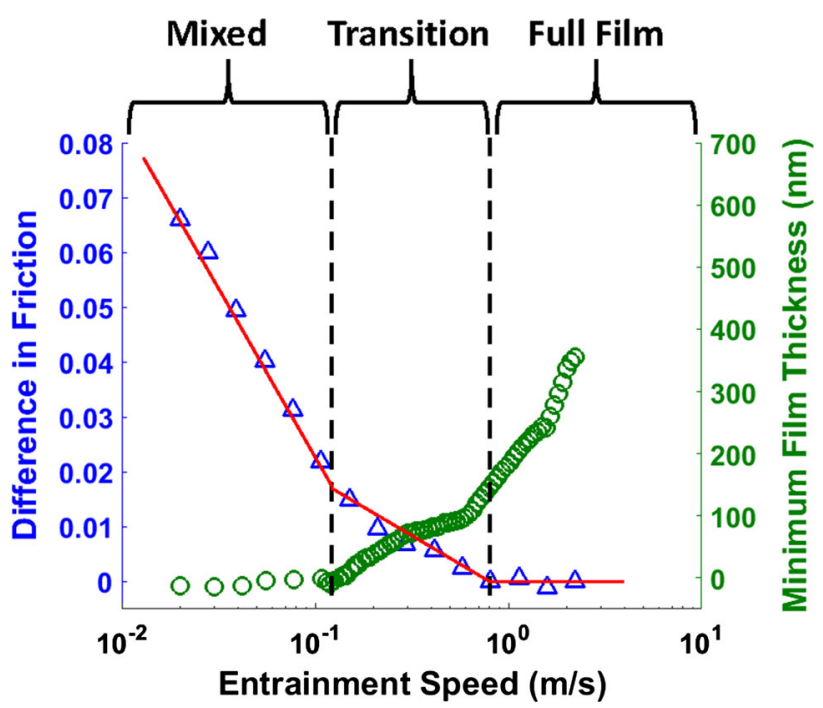

Fig. 20 Reproduction of Fig. 18b now illustrating the transition between the mixed and full-film lubrication regime and how the rate of friction variation is affected

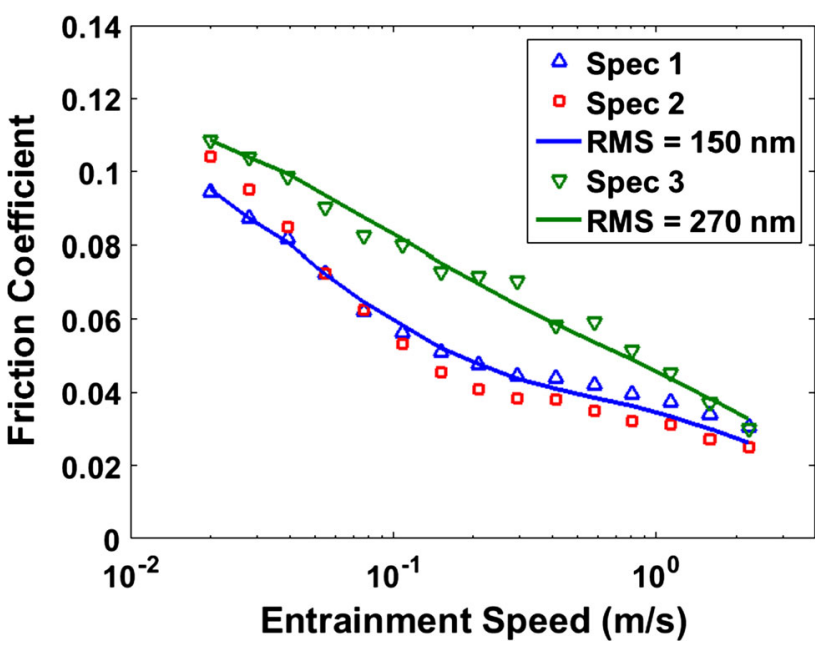

Fig. 21 Measured Stribeck curves at $50 \%$ with T68 oil together with the predicted friction curves using Eq. (8) with $\mu_{\mathrm{b}}=0.14$ and $m=2.5$ (solid line) 


\section{Conclusions}

This paper studies the influence of surface roughness on friction in a lubricated, rolling/sliding, ball-on-disc contact. Desired surface roughness structures were generated on the ball surface using a carefully controlled surface finishing process and consist of ridges parallel to the rolling direction which can be described by the dominant wavelength and peak-to-valley height. Roughnesses with different peak-to-valley heights and wavelengths were studied over a range of slide-roll ratios, entrainment speeds and with two oils with quite different viscosities, enabling experiments to be conducted over a wide range of lambda values. For comparison purposes, friction with very smooth specimens (RMS 20-30 nm) was also studied.

The tests with smooth specimens revealed the following frictional trends:

- At low $\lambda$, the friction started high and decreased with rising entrainment speed and thus film build-up. This was interpreted as the contact moving from mixed lubrication towards EHD lubrication.

- For $\lambda>5$, the friction followed the friction predicted by the Eyring model but started deviating from it at higher shear rates. This was well explained by shear heating of the lubricant film in the contact that decreased the effective viscosity and thus the friction.

The friction produced by roughened specimens was measured under the same operating conditions as the smooth specimens. Observed frictional trends were then related to the EHD film-forming behaviour of the same roughnesses, which was studied in detail in an earlier, related work [3]. Results show:

- Under the conditions studied, the rough specimens generally generate more friction than the smooth ones. This is related to lower minimum film thickness and higher pressure at the asperity peaks.

- Friction was not affected by the wavelength of the roughness, but was strongly dependent on the peak-tovalley height. Similar roughness influences on film thickness were observed in previous, related work [13].

- When the film thickness becomes sufficiently thick, the friction tends towards the friction produced at the same speed by a smooth specimen. Thus, the effect of the roughness vanishes when the EHD film is thick enough.

- The friction can be separated into a "smooth" component equal to the friction produced by a smooth specimen under the same operating conditions and a "rough" component. This rough component is independent of the SRR at the conditions tested, which suggests either some extra solid-to-solid contact or that the lubricant at the asperities reaches a limiting shear stress or both.

- For $\lambda$ between 0.8 and 3, the rough surfaces continue to give higher friction than the smooth ones even when optical interferometry indicates that there is no remaining solid-solid contact. This supports the hypothesis that the micro-EHD conditions at tops of asperity peaks are an important contributor to the overall friction in rough lubricated contacts.

- The "rough" component of friction is well described by the prevailing lambda ratio for all roughnesses and oils tested. A very simple empirical equation using the lambda ratio, boundary friction coefficient and only one fit parameter was found to predict the friction measured with all rough specimens very well.

Acknowledgments The authors wish to thank Dr. Stefan Lammens, Director SKF Engineering \& Research Center, for his kind permission to publish this article. The authors also would like to thank Petra de Bruijn for the skilful execution of the surface finishing process of the ball samples used in this work.

Open Access This article is distributed under the terms of the Creative Commons Attribution 4.0 International License (http://crea tivecommons.org/licenses/by/4.0/), which permits unrestricted use, distribution, and reproduction in any medium, provided you give appropriate credit to the original author(s) and the source, provide a link to the Creative Commons license, and indicate if changes were made.

\section{References}

1. Choo, J.W., Olver, A.V., Spikes, H.A., Dumont, M.-L., Ioannides, E.: The influence of longitudinal roughness in thin-film, mixed elastohydrodynamic lubrication. Tribol. Trans. 49, 248-259 (2006)

2. Lubrecht, A.A., Ten Napel, W.E., Bosma, R.: The influence of longitudinal and transverse roughness on the elastohydrodynamic lubrication of circular contacts. J. Tribol. 110, 421-426 (1988)

3. Guegan, J., Kadiric, A., Spikes, H.: A study of the lubrication of EHL point contact in the presence of longitudinal roughness. Tribol. Lett. 59, 1-18 (2015)

4. Lafountain, A.R., Johnston, G.J., Spikes, H.A.: The elastohydrodynamic traction of synthetic base oil blends. Tribol. Trans. 44, 648-656 (2001)

5. Bair, S., Winer, W.: Regimes of traction in concentrated contact lubrication. J. Lubr. Technol. 104, 382-386 (1982)

6. Evans, C.R., Johnson, K.L.: The influence of surface roughness on elastohydrodynamic traction. Proc. Inst. Mech. Eng. 201, 145-150 (1987)

7. Nanbu, T., Chiba, N., Kano, M., Yasuda, Y., Ushijima, K.: The effect of surface texture on traction performance in a traction drive. JSAE Rev. 24, 135-140 (2003)

8. Jefferis, J.A., Johnson, K.L.: Traction in elastohydrodynamic contacts. Proc. Inst. Mech. Eng. 182, 281-291 (1967)

9. Nanbu, T., Yasuda, Y., Ushijima, K., Watanabe, J., Zhu, D.: Increase of traction coefficient due to surface microtexture. Tribol. Lett. 29, 105-118 (2007) 
10. Johnson, K.L., Spence, D.I.: Determination of gear tooth friction by disc machine. Tribol. Int. 24, 269-275 (1991)

11. Jackson, A., Cameron, A.: An interferometric study of the EHL of rough surfaces. ASLE Trans. 19, 50-60 (1976)

12. Britton, R.D., Elcoate, C.D., Alanou, M.P., Evans, H.P., Snidle, R.W.: Effect of surface finish on gear tooth friction. J. Tribol. 122, 354-360 (2000)

13. Jacod, B., Venner, C.H., Lugt, P.M.: Influence of longitudinal roughness on friction in EHL contacts. J. Tribol. 126, 473-481 (2004)

14. Spikes, H.A., Cann, P.M.: The development and application of the spacer layer imaging method for measuring lubricant film thickness. Proc. Inst. Mech. Eng. I J. Eng. Tribol. 215, 261-277 (2001)

15. Lu, X., Khonsari, M.M., Gelinck, E.R.M.: The Stribeck curve: experimental results and theoretical prediction. J. Tribol. 128, 789-794 (2006)
16. Spikes, H.A., Jie, Z.: History, origins and prediction of elastohydrodynamic friction. Tribol. Lett. 56, 1-25 (2014)

17. Archard, J.F.: The temperature of rubbing surfaces. Wear 2 , 438-455 (1958)

18. Spikes, H.A., Zhang, J.: Reply to the Comment by Scott Bair, Philippe Vergne, Punit Kumar, Gerhard Poll, Ivan Krupka, Martin Hartl, Wassim Habchi, Roland Larson on 'History, origins and prediction of elastohydrodynamic friction' by Spikes and Jie in Tribology Letters. Tribol. Lett. 58, 1-6 (2015)

19. Johnson, K.L., Greenwood, J.A.: Thermal analysis of an Eyring fluid in elastohydrodynamic traction. Wear 61, 353-374 (1980)

20. Olver, A.V., Spikes, H.A.: Prediction of traction in elastohydrodynamic lubrication. Proc. Inst. Mech. Eng. I J. Eng. Tribol. 212, 321-332 (1998) 